\title{
Origin of two extreme solar particle events
}

\author{
Leon Kocharov, ${ }^{a}$ Alexander Mishev ${ }^{*}$, S. Pohjolainen ${ }^{c}$, M.J. Reiner ${ }^{d}$, Jeongwoo \\ Lee $^{e f}$, T. Laitinen ${ }^{g}$, L.V. Didkovsky ${ }^{h}$, V.J. Pizzo ${ }^{i}$, Roksoon Kim $^{j}$, A. Klassen ${ }^{k}, \mathbf{M}$. \\ Karlicky $^{l}$, Kyung-Suk Cho ${ }^{j m}$, G.A. Kovaltsov ${ }^{f}$, I.G. Usoskin ${ }^{a b}$, E. Valtonen ${ }^{n}$ and R. \\ Vainio $^{n}$ \\ ${ }^{a}$ Sodankylä Geophysical Observatory (Oulu unit), University of Oulu, Finland \\ ${ }^{b}$ Space Climate Research Unit, University of Oulu, Oulu FI-90014, Finland \\ ${ }^{c}$ Tuorla Observatory, University of Turku, Piikkiö FI-21500, Finland \\ ${ }^{d}$ Catholic University of America, Washington DC and NASA/Goddard Space Flight Center, USA \\ ${ }^{e}$ Department of Physics and Astronomy, Seoul National University, Seoul 151-747, Korea \\ ${ }^{f}$ A.F.Ioffe Physical-Technical Institute, Saint-Petersburg 194017, Russia \\ ${ }^{g}$ Jeremiah Horrocks Institute, University of Central Lancashire,Preston PR1 2HE, UK \\ ${ }^{h}$ University of Southern California Space Sciences Center,Los Angeles CA 90089, USA \\ ${ }^{i}$ NOAA Space Weather Prediction Center, Boulder CO 80305, USA \\ ${ }^{j}$ Korea Astronomy and Space Science Institute, Daejeon 305-348, Korea \\ ${ }^{k}$ Inst. für Experimentelle und Angewandte Physik, Christian Albrechts Universität, Kiel, Germany \\ ${ }^{l}$ Astronomical Institute of the Czech Academy of Sciences, Ondřejov 251 65, Czech Republic \\ ${ }^{m}$ University of Science and Technology, Daejeon 305-348, Korea \\ ${ }^{n}$ Space Research Laboratory, University of Turku, Turku FI-20014, Finland \\ E-mail: leon.kocharoveoulu.fi, alexander.mishev@oulu.fi,
}

\begin{abstract}
We performed an analysis of high-energy particle emission from the Sun in two extreme solar particle events observed even with ground-based neutron monitors (NMs). We model particle transport and interactions from near-Sun source through the solar wind and the Earth's magnetosphere and atmosphere in order to make a deep analysis of the events. The time profile of the proton source at the Sun is deduced and compared with observed electromagnetic emissions. Several complementary to each other data sets are studied jointly with the broadband dynamic radio spectra, EUV images as well as other data available for both events. We find a common scenario for both eruptions, including the flare's dual impulsive phase, the coronal mass ejection (CME)-launch-associated burst and the late low-frequency type III radio bursts at the time of the relativistic proton injection into the interplanetary medium. The analysis supports the idea that the two considered events start with emission of relativistic protons previously accelerated during the flare and CME launch, then trapped in large-scale magnetic loops and later released by the expanding CME.
\end{abstract}

35th International Cosmic Ray Conference - ICRC 2017-

10-20 July, 2017

Bexco, Busan, Korea

${ }^{*}$ Speaker. 


\section{Introduction}

Thu study of high energy solar particles events following solar eruptions is important in order to understand their acceleration at the Sun as well as their transport in the interplanetary medium $[1,2,3]$. A major solar eruption comprises both a gradual solar flare, observed in X-rays and other electromagnetic emissions, and a wide and fast CME. There are evidences that the highenergy $(\geq 50 \mathrm{MeV}$ ) protons can be produced by both flare and CME, while relative contributions of the eruption stages to particles arriving at 1 au are still under discussion [4, 5, 6]. Detection of solar protons of above $0.4 \mathrm{GeV} /$ nucl produced in strong events is possible with ground-based detectors as NMs, when the energy of the solar energetic particle (SEP) is enough high to produce an atmospheric cascade. The ground level enhancement (GLE) events are considered extreme [7] and occur roughly one per year with greater probability during maximum and decline phase of the solar activity cycle [8]. Modern space-borne instruments can measure high-energy nuclei with a good resolution in both energy and arrival direction, but due to their low orbits, they suffer regular gaps in the solar particle observations.

As example, SOHO carries a set of solar telescopes and coronographs in addition to particle detectors. Hence, the about 20 years of SOHO operation have made it possible the multi-wavelength observation a common practice. Nowadays, it is usual to compare the timing of the "first particle" emission from the Sun with observed electromagnetic emissions. However, such consideration could not be sufficient, because in the major (gradual) SEP events, the particle injection from the Sun is a prolonged process. Therefore, the entire time profile of the SEP source should be considered in order to build a clear picture. For the present study, we have selected two apparently similar GLEs, namely GLE 56 on 2 May 1998 and GLE 67 on 2 November 2003. Here, we perform an analysis of particle data and corresponding particle transport modelling, from the Sun to a detector on the ground, in order to deduce the particle source profile at the Sun. Then, the inferred source profile is compared to the multi-wavelength data of solar observations, hence a possible solar origins of the extreme particle events are investigated.

\section{Observations and modelling}

We consider two events: GLE 56 on 2 may 1998 and GLE 67 on 2 November 2003. Those events were moderately strong, with a step-like onset delayed with respect to the impulsive phase of the corresponding flare. Figure 2 illustrates the associated active regions and structures. On 2 May 1998 the flare was not far from the disk center, (region A in Fig.1), and the active region was interconnected (N25E25; region B) by a large-scale loop structure that was later observed to form part of the CME. Accordingly the 2 November 2003 flare was closer to west limb, at S14W56 in AR10486. Coronal dimming areas, seen in the EIT difference images between regions A and B, are footprints of departing CMEs. The two eruptions are qualitatively similar to each other, but they strongly differ in the total energetics. The GLE 56 is associated with the X1.1/3B solar flare and moderately fast CME, while the GLE 67 is associated with the X8.3/2B solar flare and very fast CME. In Fig. 1 are shown the EUV images before the eruption, where the flaring active regions are labelled with A. Coronal dimming area is seen between the active regions A and B. On 2003 November 2, the dimming is seen also above the west limb and near the south pole. 


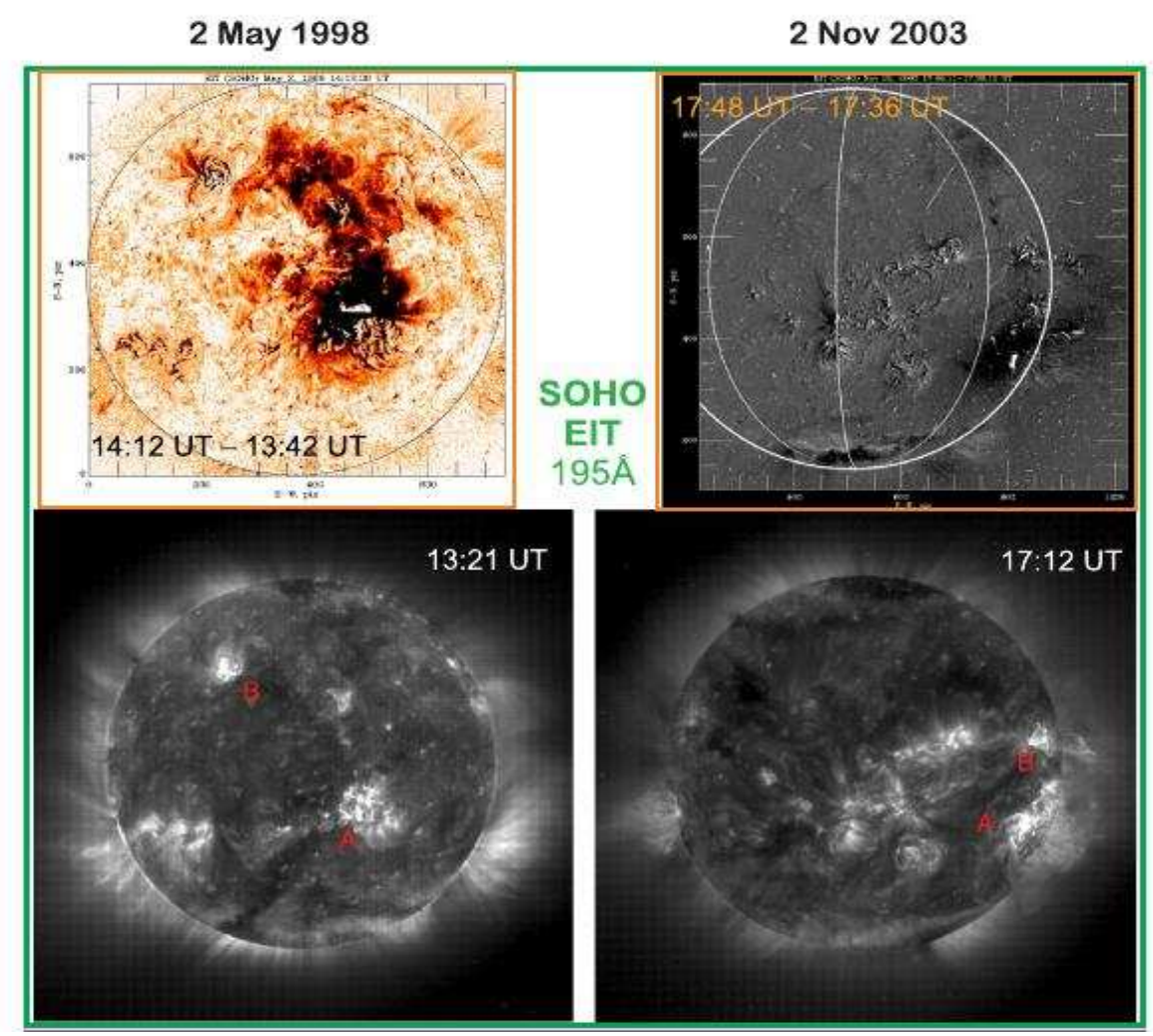

Figure 1: Global structure of the two eruptions as observed by SOHO/EIT. The upper row depicts the difference images revealing the post-eruption dimming areas, while the second row depicts the EUV images before the eruption. The flaring active regions are labelled with A. Coronal dimming area is seen between the active regions $\mathrm{A}$ and $\mathrm{B}$.

Accordingly the time profiles of the two soft X-ray flares, CMEs and GLEs are compared in Fig.2. Here, we have selected for each event a low rigidity cut-off polar NM that reveals the earliest rise of the count rate. In the considered event pair, the time delays between the flare impulsive phase (steep rise of the soft X-ray emission), on the one hand, and the CME arrival at a particular altitude and the GLE rise time at the Sun, on the other hand, are organized according to the reciprocal of an average speed of CME.

The most important is that neither re-scaling would result in the profile coincidence, if the reference time was taken not at the flares's impulsive phase. In the two considered events, the flare pulse precedes CME, being a kind of triggering event. Thus, according to shown measurements on Fig. 1 and Fig. 2 the two eruptions are morphologically homologous.

The comparison of the energetic particle production with the associated solar electromagnetic emissions is necessary to infer from the 1 au observations of the particle source profile at the Sun. Generally, the source profile can be derived by fitting the observed particle time-intensity profile and pitch angle distribution with a particle injection and interplanetary transport model. However, the GLE 56 is very anisotropic event and the interplanetary transport modelling can be replaced with a simple shifting of the 1 au profile back to the Sun considering the flight time appropriate to the particle energy. The time shifting method can reproduce the rise and the maximum phase 


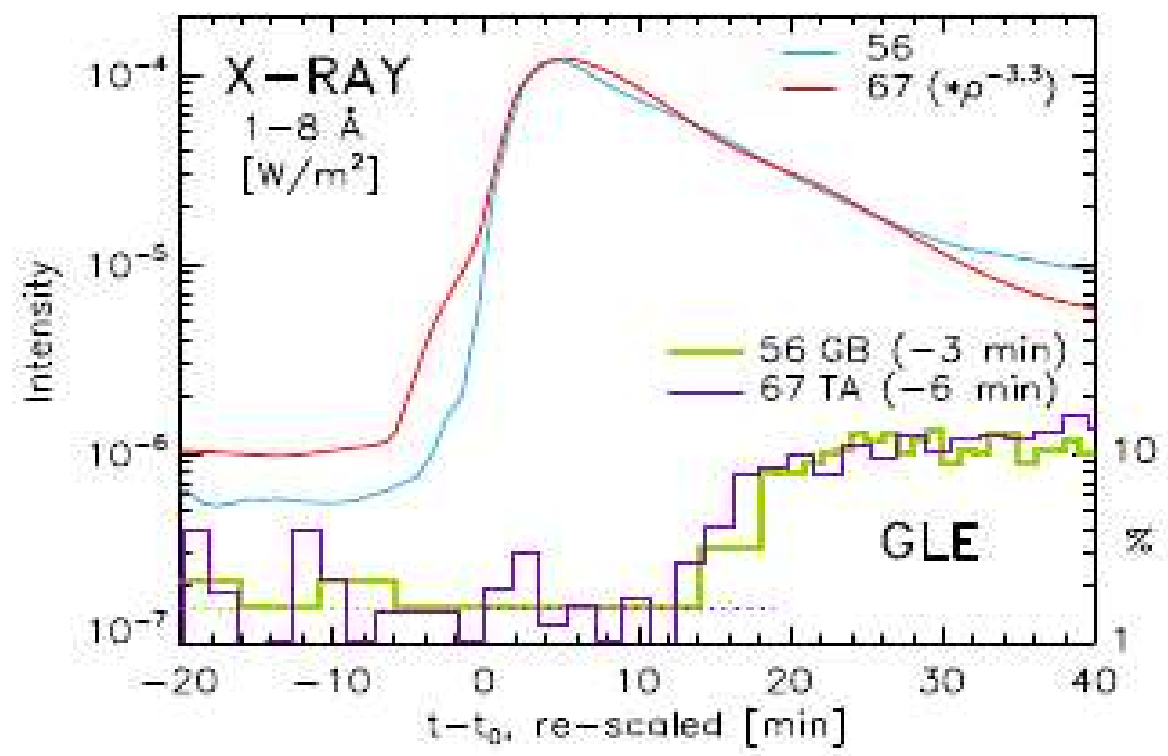

Figure 2: Time profiles of the NM count rate (GLE) and soft X-ray flare. The timescale of GLE 67 event is either stretched out with scaling factor $r=1.8$, for CME and GLE, or compressed with the same scaling factor, for the soft X-ray flare. The abbreviations GB and TA correspond to Goose Bay and Terre Adelie NMs. For a comparison with the 1 au observed electromagnetic emissions, timing of each GLE is corrected for a difference of the Sun-Earth transport time between protons and photons the correction depends on the interplanetary transport conditions in a particular event.

of the solar source in strongly anisotropic events but shall not be used for the decay phase [9]. Accordingly the GLE 67 was characterized by a significant anisotropy as observed by global NM network (Oulu NM count rate rose early in the event, despite the anti-Sun viewing direction). Obviously, the proton angular distribution was not very narrow and hence, the modelling of the particle transport from the solar source to 1 au is performed, the details are given elsewhere [10]. The summary of the electromagnetic observations is presented in details in [10]. The SEP spectral and angular characteristics are derived using global NM network data and convenient optimization method [11, 12], the details are given in this volume and in [10].

\section{Discussion}

At recent was found that mostly all of the proton events are preceded by type III bursts and all are preceded by CMEs, where the open field lines extend from within $0.5 R_{\odot}$ into the interplanetary medium and the burst-generating electrons originate from the reconnection regions below fast CMEs [13]. However, it was shown that they typically do not coincide and the high-energy protons and the type III electrons could not originate from one and the same source [14]. Here, we perform analysis of the entire time profile of the SEP source. We have deduced the source profile of the $>400 \mathrm{MeV} / \mathrm{n}$ protons leading to a GLE. The type III bursts occurred when CME reaches the top sections of highest loops and expands in the lateral direction. Hence, the magnetic reconnection with open filed that is responsible for the type III emission could occur at the CME flank. 
The impulsive phases of both flares comprise two pulses, F1 and F2 (Fig.3, 4). The sources are situated at close but still different locations and both are rich in hard X-rays as compared to microwaves. The first pulse F1 triggers the Moreton wave that either causes or just visualizes the eruption widening. After F1, both eruptions develop in a similar way but with different rates. At recent was shown a statistical evidence that large-scale coronal eruptions associated with EIT waves exhibit characteristics that are consistent with a blast wave originating from a localized region [15]. There is theoretical evidence that in fast CMEs, flare reconnection is the primary mechanism responsible for both flare and CME [16]. After the second pulse, F2, the soft X-ray flare has reached its maximum and further develops as a gradual. At the time of the soft X-ray maximum, the microwave-rich burst $\mathrm{C} 1$ starts to rise and the source is situated beneath the $\mathrm{CME}$ and outside of active region.

A CME appears at distance of about $1.7 R_{\odot}$ and then accelerates in the radial direction and the $\mathrm{C} 1$ burst coincides with the main acceleration period. Thus $\mathrm{C} 1$ is regarded as a signature of the launch of the wide CME located above it, namely it is part of the CME launch system. Accordingly on 2 November 2003, when the $\mathrm{CME}$ nose reaches $2.3 R_{\odot}$ the $\mathrm{CME}$ also starts a lateral expansion. Hence, the accelerated particles are released from the largest loop-like magnetic structures after the CME-driven magnetic reconnection with open magnetic field at the CME flank and/or at tips of helmet streamers.

Thus, we speculate that those protons are released at magnetic reconnection between the CMEloops originating from the flare region ( $\mathrm{CME}$ core) and the south-west streamer, as visualized by the brightening $(\mathrm{J})$. We suggest that the high-energy particles are produced in the flare, then get trapped in the CME core and finally escape after magnetic reconnection between the CME core and open field. According our analysis of GLE 67 spectral features, the observed delays between the flare, the CME launch, and the GLE make it possible for the initially accelerated SEPs to be re-accelerated during the trapping in coronal and CME loops $[17,18]$.

\section{Conclusion}

We consider data of multi-wavelength observations carried out by different instruments of extreme solar particle events, where SEPs are accelerated to relativistic energies and are observed even in the ground-based NMs. We consider a pair of extreme particle events, the events of 2 May 1998 (GLE 56) and 2 November 2003 (GLE 67). We performed an analysis of the data of particle and electromagnetic observations of the two GLEs. For the first time, we have deduced the time profile of the relativistic proton source at the Sun and compared it with data of electromagnetic observations including both the dynamic spectra and images. After a detailed analysis of the global morphology of involved active regions, magnetic field structures, and CMEs reveals a qualitative similarity between the two eruptions. Time profiles of the soft X-ray flare, the CME height, and the relativistic proton emission of one eruption coincide with corresponding profiles of another eruption after a rescaling of time according to the CME speed, with the flares's first major pulse being the eruption triggering event, and the soft X-ray flare magnitude scaled as a power of the CME speed. We find a common scenario for both GLE-producing eruptions, which includes the flare's dual impulsive phase, followed by the CME launch. 


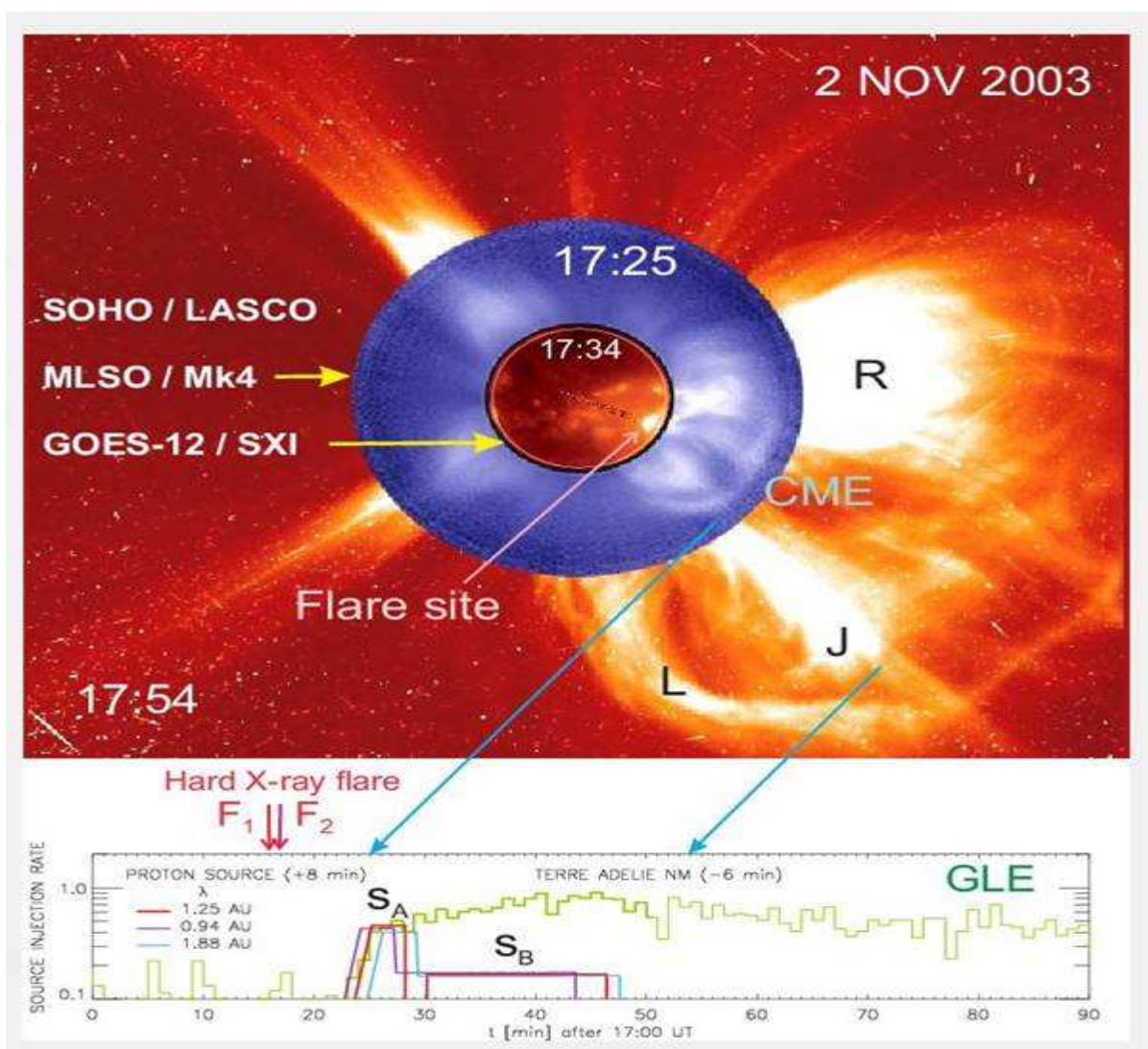

Figure 3: Evolution of GLE 67 after 17:25 UT. The rope structure (R) exists already in the low corona. The jet-like structure (J) appears after 17:30 UT at the location of previous narrow, low ejections along the streamer.

Two major pulses of the flares's impulsive phase are strong in both hard X-rays and microwaves. The first pulse causes a global coronal wave. The wave could trigger, in particular, a compact, microwave-rich burst $\mathrm{C} 1$ situated outside the flare active region. The $\mathrm{C} 1$ burst coincides with the main acceleration period of $\mathrm{CME}$ in the low corona. We identify the decimetric-continuum burst $\mathrm{C} 1$ with a CME launch event. Particle transport from Sun to Earth in the GLE 56 event was essentially not free of scattering and, therefore, the transport modelling shall be applied to deduce the time profile of the relativistic proton injection at the Sun. First emission of relativistic protons from the Sun begins when CME reaches the tips of helmet streamers. In the GLE 67 the first pulse of the proton emission also coincides with a well-observed period of fast lateral expansion of CME. The second period of the proton emission may be ascribed to the interaction of the CME core with the coronal streamer. The proton emissions coincide with a series of the low-frequency type III radio bursts, indicative of the magnetic reconnection with open magnetic field lines, driven by the rising CME. According our analysis the observations supports the idea that the two considered events start with emission of relativistic protons previously accelerated at the flare and separately at the CME launch, then trapped in large-scale magnetic loops, possibly re-accelerated there by shock and turbulence, and later released by the expanding CME via the CME-induced magnetic reconnection with open field. 


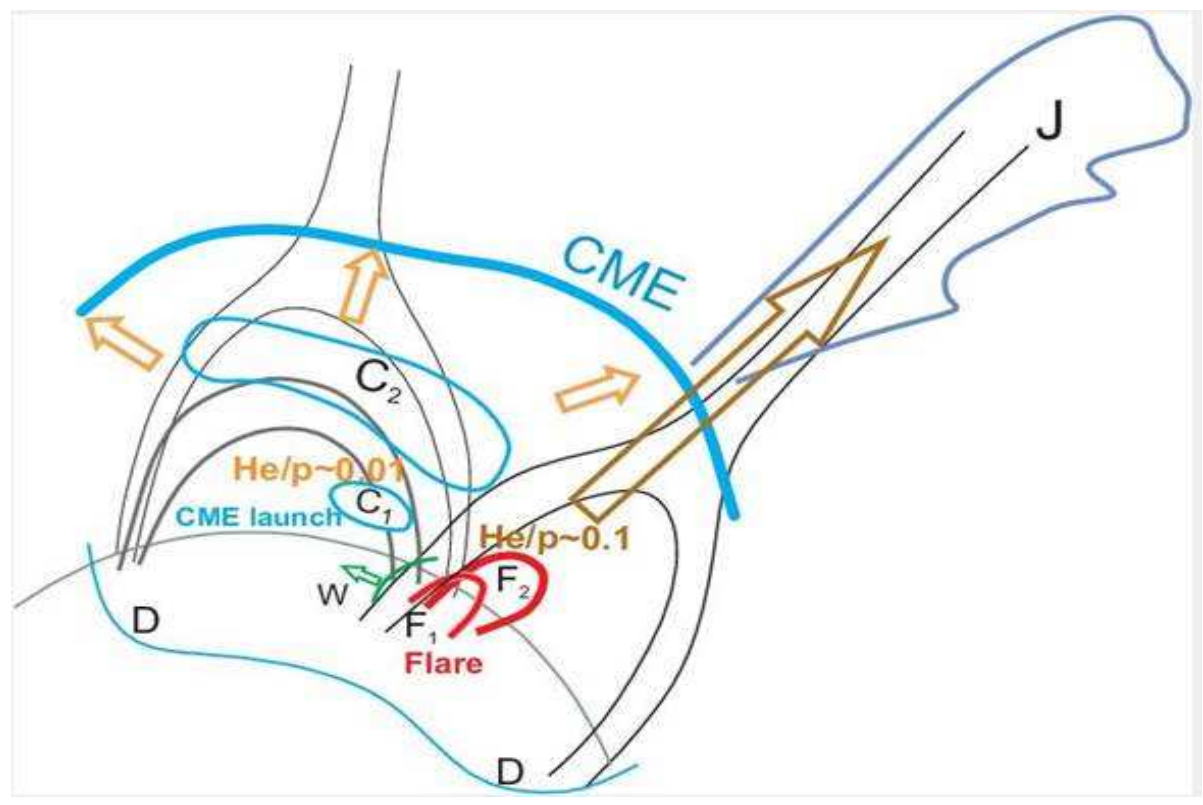

Figure 4: Eruption scenario for the two considered flare-CME-GLE events flare. The impulsive phase of comprises two outbursts, F1 and F2. The first flaring causes the global coronal wave (W). The radio burst $\mathrm{C} 1$ is associated with a CME-launch event, which may be triggered by the wave. The EUV dimming (D) and the extended radio source (C2) are produced by the rising CME. Injection of protons into the solar wind occurs upon the CME arrival at the top sections of helmet streamers and concurrently with the lateral expansion of CME at its right flank. Interaction ( $\mathrm{J}$ ) between the CME loops originating from the flare region and the streamer is responsible for the second, more prolonged, emission. Protons escape into the interplanetary medium at the CMEdriven magnetic reconnection with an open field.

\section{Acknowledgements}

This study was supported by the Academy of Finland projects 260596, 258963, and 272157 (Center of Excellence ReSoLVE). A.K. was supported by the German DLR under grant 50 OC 1302, J.L. was supported by the BK21 Plus Program (21A20131111123) funded by the Ministry of Education (MOE, Korea) and National Research Foundation of Korea (NRF), M.K. was supported from Grant P209/12/0103 of the Grant Agency of the Czech Republic. We acknowledge the NMDB (www.nmdb.eu) and the PIs of NM stations used for the analysis of GLEs.

\section{References}

[1] H. Debrunner, E. Flückiger, H. Gradel, J. Lockwood and R. McGuire, Observations related to the acceleration, injection, and interplanetary propagation of energetic protons during the solar cosmic ray event on February 16, 1984, Journal of Geophysical Research 93 (1988), no. A7 7206-7216.

[2] J. A. Lockwood, H. Debrunner and E. O. Flükiger, Indications for diffusive coronal shock acceleration of protons in selected solar cosmic ray events, Journal of Geophysical Research: Space Physics 95 (1990), no. A4 4187-4201. 
[3] D. Reames, Particle acceleration at the sun and in the heliosphere, Space Sci. Rev. 90 (1999), no. 3-4 413-491.

[4] E. Cliver, S. Kahler and D. Reames, Coronal shocks and solar energetic proton events, Astrophysical Journal 605 (2004) 902-910.

[5] D. Reames, The two sources of solar energetic particles, Space Sci. Rev. 175 (2013), no. 1-4 53-92.

[6] M. Desai and J. Giacalone, Large gradual solar energetic particle events, Living Reviews in Solar Physics 13 (2016), no. 13.

[7] N. Gopalswamy, S. Yashiro, N. Thakur, P. Mäkelä, H. Xie and S. Akiyama, The 2012 July 23 backside eruption: an extreme energetic particle event?, Astrophysical Journal 833 (2016), no. 2216.

[8] M. Shea and D. Smart, A summary of major solar proton events, Solar Physics 127 (1990) 297-320.

[9] L. Kocharov, A. Klassen, E. Valtonen, I. Usoskin and J. Ryan, Comparative morphology of solar relativistic particle events, Astrophysical Journal Letters 811 (2015), no. 1 L9.

[10] L. Kocharov, S. Pohjolainen, A. Mishev et al., Investigating the origins of two extreme solar particle events: Proton source profile and associated electromagnetic emissions, The Astrophysical Journal 839 (2017), no. 279.

[11] A. Mishev, L. Kocharov and I. Usoskin, Analysis of the ground level enhancement on 17 May 2012 using data from the global neutron monitor network, Journal of Geophysical Research 119 (2014) $670-679$.

[12] A. Mishev and I. Usoskin, Analysis of the ground level enhancements on 14 July 2000 and on 13 December 2006 using neutron monitor data, Solar Physics 291 (2016), no. 4 1225-1239.

[13] H. Cane, W. Erickson and N. Prestage, Solar flares, type iii radio bursts, coronal mass ejections, and energetic particles, Journal of Geophysical Research: Space Physics 107 (2002), no. A10 1315.

[14] N. Gopalswamy, H. Xie, S. Yashiro, S. Akiyama, P. Mäkelä and I. Usoskin, Properties of ground level enhancement events and the associated solar eruptions during solar cycle 23, Space Science Reviews 171 (2012), no. 1-4 23-60.

[15] T. Howard and V. Pizzo, Challenging some contemporary views of coronal mass ejections. $i$. the case for blast waves, Astrophysical Journal 824 (2016), no. 292.

[16] J. Karpen, S. Antiochos and C. Devore, The mechanisms for the onset and explosive eruption of coronal mass ejections and eruptive flares, Astrophysical Journal 760 (2012), no. 181.

[17] L. Kocharov, R. Vainio, J. Pomoell, E. Valtonen, A. Klassen and C. Alex Young, Non-standard energy spectra of shock-accelerated solar particles, Astrophysical Journal 753 (2012), no. 187.

[18] A. Afanasiev, R. Vainio and L. Kocharov, The effect of stochastic re-acceleration on the energy spectrum of shock-accelerated protons, Astrophysical Journal 790 (2014), no. 136. 